\title{
BMJ
}

\section{Novel approach to antibiotic prophylaxis in percutaneous endoscopic gastrostomy (PEG): randomised controlled trial}

\author{
John Blomberg, PhD student,,2 Pernilla Lagergren, assistant professor, '. Lena Martin, postdoctoral scientist, \\ Fredrik Mattsson, statistician, ${ }^{1}$ Jesper Lagergren, professor $^{1,3}$
}

${ }^{1}$ Upper Gastrointestinal Research, Department of Molecular Medicine and Surgery, Karolinska Institutet, SE-171 76 Stockholm, Sweden

${ }^{2}$ Department of Surgery, Gastrocenter, Karolinska University Hospital, SE-141 86 Stockholm, Sweden

${ }^{3}$ King's College, London Correspondence to: J Blomberg john.blomberg@karolinska.se

Cite this as: $B M J$ 2010;340:c3115 doi:10.1136/bmi.c3115

\section{ABSTRACT}

Objective To evaluate a new and simpler strategy of antibiotic prophylaxis in percutaneous endoscopic gastrostomy (PEG).

Design Single centre, two arm, randomised, controlled, double blind clinical trial.

Setting Endoscopy unit in Karolinska University Hospital, Stockholm, Sweden, between 3 June 2005 and 31 October 2009.

Participants 234 patients with an indication for PEG who gave informed consent to participate.

Intervention A single $20 \mathrm{ml}$ dose of the oral solution of sulfamethoxazole and trimethoprim (also known as co-trimoxazole or Bactrim; F Hoffmann-La Roche Ltd, Basel, Switzerland) deposited in the PEG catheter immediately after insertion. The control group received standard prophylaxis consisting of a single intravenous dose of $1.5 \mathrm{~g}$ cefuroxime (Zinacef; GlaxoSmithKline, London) administered before insertion of the PEG tube. Main outcome measure Primary outcome was the occurrence of clinically evident wound infection within 14 days after insertion of the PEG catheter. Secondary outcomes were positive bacterial culture and blood tests (highly sensitive $C$ reactive protein and white blood cell count). All randomised patients were included in an intention to treat analysis.

Results Of the 234 patients included in this study, 116 were randomly assigned to co-trimoxazole and 118 to cefuroxime. At follow-up 7-14 days after insertion of the PEG catheter, wound infection was found in 10 (8.6\%) patients in the co-trimoxazole group and 14 (11.9\%) in the cefuroxime group, which corresponds to a percentage point difference of $-3.3 \%$ (95\% confidence interval $-10.9 \%$ to $4.5 \%$ ). The per protocol analysis, which comprised 100 patients in each group, gave similar results-10\% and $13 \%$ infection in the co-trimoxazole and cefuroxime groups, respectively (percentage point difference $-3.0 \%, 95 \% \mathrm{Cl}-11.8 \%$ to $5.8 \%$ ). Both these analyses indicate non-inferiority of co-trimoxazole compared with cefuroxime because the upper bounds of the confidence intervals are lower than the pre-determined non-inferiority margin of $15 \%$. Analyses of the secondary outcomes supported this finding. Conclusion $20 \mathrm{ml}$ of co-trimoxazole solution deposited in a newly inserted PEG catheter is at least as effective as cefuroxime prophylaxis given intravenously before PEG at preventing wound infections in patients undergoing PEG. Trial registration Current Controlled Trials ISRCTN18677736.

\section{INTRODUCTION}

Percutaneous endoscopic gastrostomy (PEG), which is mainly used to maintain enteral nutrition in patients with longstanding eating difficulties, has, since its introduction in $1980,{ }^{1}$ steadily increased in use to become a common intervention. As a consequence of malnutrition and comorbidities, patients receiving a PEG catheter are often vulnerable to infection, ${ }^{2}$ which is the main acute problem after insertion of the PEG catheter. ${ }^{3}$

Infection at the insertion site occurs in about one third of all patients who receive a PEG catheter, ${ }^{4-8}$ and such infections are often difficult to treat. Meta-analyses of randomised trials have established that wound infection rates decrease substantially when intravenous antibiotic prophylaxis is given before the PEG procedure. ${ }^{9-12} \mathrm{~A}$ single intravenous dose of the cephalosporin cefuroxime (Zinacef; GlaxoSmithKline, London), given one hour before the PEG procedure, is well established as the gold standard method of antibiotic prophylaxis and is recommended by the British Society of Gastroenterology (evidence grade Ia and recommendation grade $\mathrm{A})^{13}$ because of its good effect on the bacteria commonly found in the upper gastrointestinal tract. ${ }^{14}{ }^{15}$ Disadvantages of this strategy include the high cost, the need to give the medication intravenously, and the need to give it before insertion of the PEG catheter, even though in about $10 \%$ of patients insertion of the catheter proves to be impossible. ${ }^{16}$

We hypothesised that there is a less expensive, technically easier, and at least as effective way of infection prophylaxis than using cefuroxime; that is, depositing a single dose of a combination of a sulphonamide and trimethoprim (also known as co-trimoxazole or Bactrim; F Hoffmann-La Roche Ltd, Basel, Switzerland), as an oral solution, directly in the newly inserted PEG catheter. To test this hypothesis we conducted a large randomised clinical trial.

\section{METHODS}

Design and intervention

We undertook a double blind, randomised clinical trial to test whether a single dose of $20 \mathrm{ml}$ co-trimoxazole 
(800 mg sulfamethoxazole and $160 \mathrm{mg}$ trimethoprim) deposited in the PEG catheter immediately after insertion was at least as good (non-inferiority assumption) for the prevention of acute wound infections as the standard antibiotic prophylaxis of a single intravenous dose of $1.5 \mathrm{~g}$ of cefuroxime administered one hour before insertion of the PEG catheter. We chose co-trimoxazole rather than an oral cephalosporin as the test drug because of the following advantages: more rapid absorption in the upper gastrointestinal tract; longer half life; doesn't need to be mixed with food intake (which is difficult immediately after PEG); lower risk of infection with Clostridium difficile ${ }^{17}$; ; and lower price.

\section{Patients}

All patients at the Karolinska University Hospital, Stockholm, Sweden, who were referred to the endoscopy unit for PEG during the period 3 June 2005 to 31 October 2009 were considered for inclusion. Those who did not meet any exclusion criteria, had no contraindications for PEG, and were able to give consent to participation in the study after receiving oral and written information were included. Reasons for exclusion were ongoing antibiotic treatment, illness too severe to allow the patient to participate, or allergy to any of the antibiotic alternatives.

\section{Randomisation and blinding}

The randomisation process was conducted by personnel at a hospital department not engaged in the care of the included patients. After a patient had agreed to participate, these personnel were contacted by telephone and on request opened a closed envelope, taken from a pre-prepared block (50 envelopes in each block) of equally distributed and mixed envelopes, containing a randomisation sheet with information on the drug to be used. The patient's personal identity number and the date were documented on the randomisation sheet. Thereafter, the sheet was sent to the study coordinator. The patient and the nurse who evaluated the patient at the follow-up visit were both blinded to which antibiotic was to be given. The blinding of the patients was accomplished by using intravenous fluid and manipulating the newly inserted PEG catheter in all patients. This sham manoeuvre was facilitated by the use of sedation. The nurses who evaluated the patients at the follow-up visit were not involved in insertion of the PEG catheter, including the administration of antibiotic prophylaxis.

\section{The PEG procedure}

All patients fasted for at least six hours before the PEG procedure. The local anaesthetic lidocaine (Xylocaine; AstraZeneca, London) was administered as a throat spray, after which midazolam (Dormicum; Roche) was given intravenously for sedation. A standard, silicon wire reinforced, 20 Charrière Bard FasTrac pull PEG catheter (Bard Norden AB, Sweden) was used in all patients. The catheter was pulled down through the mouth, into the stomach and out through the abdominal wall, and anchored with an external bolster. The tightness of the bolster position was checked by gently pulling on the catheter to see if the bolster could easily be lifted about $5 \mathrm{~mm}$ from the abdominal wall, thus ensuring that the fit was not too tight. The PEG catheter could then be used for infusion within two hours.

Patients were given an information folder that contained a description of what had been done during the procedure, the brand name of the PEG catheter, instructions on how to perform daily care of the catheter and the wound site, solutions to common problems associated with PEG, general nutritional advice, and a telephone number for the outpatient surgical clinic and a dietitian for contact whenever advice about the PEG device or nutrition was needed.

\section{Data collection}

The baseline data collected were name, personal identity number, age, sex, indication for PEG, comorbidities, and tobacco smoking habits. Peripheral blood samples were collected for measurement of haemoglobin, highly sensitive $\mathrm{C}$ reactive protein, white blood cells, and albumin. Objective measurements of weight and height were collected for assessment of body mass index (BMI).

At the follow-up appointment within 7-14 days of insertion of the PEG catheter, any visible signs of infection, leakage, diarrhoea, constipation, abdominal pain, and fever were documented and treated if necessary. A sample for bacterial culture was retrieved from the PEG stoma and blood samples were collected for measurement of the same parameters as presented above. If the patient was unable to attend the follow-up visit, the patient or caring personnel were contacted by telephone and interviewed by the study nurse, who asked whether any complications had occurred. If this interview led to any suspicion of a complication, the patient was given a new urgent appointment and was checked up at the outpatient clinic, where any infectious complications were documented.

Finally, information on any deaths was obtained by checking the complete and continuously updated Swedish Total Population Register.

\section{Outcome assessment}

The primary outcome was occurrence of a clinically identifiable wound infection, as judged by a red zone around the catheter or occurrence of pus, subcutaneous swelling, and pain on palpation in the area around the catheter. Secondary outcomes were objective signs of infection, including a positive bacterial culture, high levels of highly sensitive $\mathrm{C}$ reactive protein, and a high white blood cell count.

\section{Statistical analysis}

The primary objective was to assess the non-inferiority of co-trimoxazole compared with cefuroxime. The difference between the co-trimoxazole and the cefuroxime group in the proportion of patients with an infection was estimated, as was the corresponding two sided 95\% confidence interval (CI). Non-inferiority of co-trimoxazole would be achieved if the upper 
limit of the $95 \%$ CI was smaller than the pre-specified non-inferiority margin of $15 \%$. The non-inferiority margin was selected on a clinical basis.

Assuming infection rates of $15 \%$ or $20 \%$ in both groups, the number of patients required to establish non-inferiority with $80 \%$ power and significance level of $5 \%$ were 89 or 112 , respectively. Differences between the two treatment groups were tested with the $t$ test or the Mann-Whitney $\mathrm{U}$ test for continuous variables, depending on which assumption was met. The $\chi^{2}$ test was used for categorical variables as long as the expected count in every cell was at least five, otherwise Fisher's test of exact probability was used.

Intention to treat was the main analysis strategy and encompassed all randomised patients irrespective of whether they received a PEG catheter or not, or were followed up or not. A per protocol analysis was conducted as a sensitivity test, and included only those patients who underwent PEG and were followed up. The software SAS Statistical Package (Version 9.2; SAS Institute Inc, Cary, NC) was used for all statistical analyses.

\section{RESULTS}

Inclusion of patients

The figure presents a flow chart of patients considered for the study. During the four year study period, 535 patients with a need for PEG were considered for inclusion. Of these patients, 301 were unable to give informed consent or did not wish to participate in the study. The remaining 234 patients were randomly assigned to a study arm and included in the intention to treat analysis: 116 patients in the co-trimoxazole group and 118 patients in the cefuroxime group. A total of $12(10 \%)$ patients in each group did not undergo PEG for anatomical reasons. Between the PEG procedure and the follow-up visit, five patients died, one patient pulled out the PEG catheter without ensuing complications, three patients were lost to follow-up,

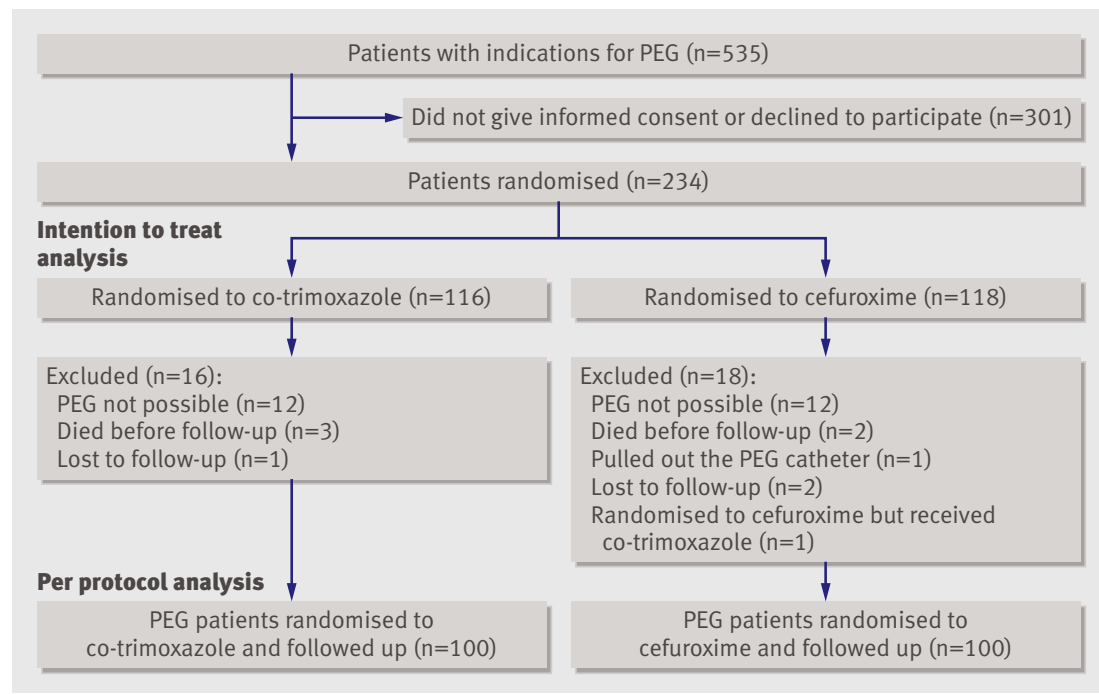

Flowchart of patients considered for the trial of antibiotic prophylaxis in patients with a need for percutaneous endoscopic gastrostomy (PEG)
Table 1|Characteristics and indications for percutaneous endoscopic gastrostomy (PEG) for the 234 patients randomised to Bactrim or Zinacef

\begin{tabular}{lcc} 
& $\begin{array}{c}\text { Co-trimoxazole } \\
(n=116)\end{array}$ & $\begin{array}{c}\text { Cefuroxime } \\
(n=118)\end{array}$ \\
Characteristic & $42(36)$ & $31(26)$ \\
\hline Female & $66(12)$ & $65(12)$ \\
\hline $\begin{array}{l}\text { Age (mean in years ( } \pm \text { standard } \\
\text { deviation) }\end{array}$ & $8(7)$ & $5(4)$ \\
\hline 30 day mortality & $32(28)$ & $41(35)$ \\
\hline Current tobacco smoker & $8(7)$ & $8(7)$ \\
\hline Diabetes & $58(50)$ & $69(59)$ \\
\hline Indication for PEG & $24(21)$ & $18(15)$ \\
\hline Ear, nose, or throat cancer & $20(17)$ & $10(9)$ \\
\hline Neurological disease & $4(4)$ & $7(7)$ \\
\hline Oesophageal cancer & $1(1)$ & $0(0)$ \\
\hline Stroke & $0(0)$ & $1(1)$ \\
\hline Dementia & $9(8)$ & $111)$ \\
\hline Gastric cancer & & \\
\hline Other & & \\
\hline Al valus & & \\
\hline
\end{tabular}

All values are number (\%) unless stated otherwise and rounded up, which in some cases gives a sum of more than $100 \%$.

and one patient who was randomised to cefuroxime received co-trimoxazole instead. After exclusion of these 34 patients, 100 patients in each group remained for the per protocol analysis.

\section{Patient characteristics}

Basic characteristics of the 234 included patients are presented in table 1. No substantial differences existed between the two groups regarding age, mortality at 30 days, tobacco smoking status, or occurrence of diabetes, but the proportion of men in the cefuroxime group was non-significantly higher. The indications for PEG were also evenly distributed, except for a higher proportion of patients with oesophageal cancer in the co-trimoxazole group. Cancer of the ear, nose, or throat was the most common reason for PEG in both groups.

\section{Complications}

Table 2 summarises the occurrence of pre-defined complications in the two treatment groups after insertion of the PEG catheters. One or more of these complications were found in $43 \%(50 / 116)$ of the patients in the co-trimoxazole group and in $47 \%(55 / 118)$ of the patients in the cefuroxime group $(\mathrm{P}=0.590)$. None of the individual complications was strongly over-represented in any of the groups, but the frequencies of catheter leakage, constipation, abdominal pain, fever, and infection were non-significantly higher in the cefuroxime group (table 2).

No adverse reactions to the antibiotics used were recorded among the included patients.

\section{Wound infection (primary outcome)}

In the intention to treat analysis, the occurrence of clinically identifiable wound infection was $8.6 \%(10 / 116)$ in the co-trimoxazole group and $11.9 \%(14 / 118)$ in the cefuroxime group. The percentage point difference between the rate of infection in the co-trimoxazole 
Table 2 |Complications after insertion of the percutaneous endoscopic gastrostomy (PEG) catheter

\begin{tabular}{lcc} 
No complications & $\begin{array}{c}\text { Co-trimoxazole } \\
(\mathrm{n}=116)\end{array}$ & $\begin{array}{c}\text { Cefuroxime } \\
(\mathrm{n}=118)\end{array}$ \\
\hline Abdominal pain & $66(57)$ & $63(53)$ \\
\hline Leakage around the catheter & $13(11)$ & $17(14)$ \\
\hline Infection & $10(9)$ & $16(14)$ \\
\hline PEG procedure failed & $10(9)$ & $14(12)$ \\
\hline Constipation & $12(10)$ & $12(10)$ \\
\hline Diarrhoea & $7(6)$ & $11(9)$ \\
\hline Patient died before follow-up & $5(4)$ & $4(3)$ \\
\hline Fever & $3(3)$ & $2(2)$ \\
\hline Patient pulled out PEG catheter & $0(0)$ & $3(3)$ \\
before follow-up & $0(0)$ & $1(1)$ \\
\hline
\end{tabular}

Values are number (\%). Each individual patient could have more than one complication.

group and that in the cefuroxime group was - $3.3 \%$ (95\% CI $-10.9 \%$ to $4.5 \%)$. The per protocol analysis had similar results-10\%(10/100) and 13\% (13/100) infection in the co-trimoxazole and cefuroxime groups, respectively (percentage point difference $-3.0 \%, 95 \% \mathrm{CI}-11.8 \%$ to $5.8 \%$ ). Both these analyses indicated non-inferiority of co-trimoxazole compared with cefuroxime because the upper bounds of the confidence intervals are lower than the pre-determined non-inferiority margin of $15 \%$.

\section{Positive bacterial culture or blood chemistry (secondary outcomes)}

Bacterial cultures were collected in $70 \%(81 / 116)$ of the patients in the co-trimoxazole group and in $71 \%$ (84/ 118 ) in the cefuroxime group. A positive bacterial culture was identified in 44 out of 81 patients $(54 \%)$ in the co-trimoxazole group and in 53 out of 84 patients $(63 \%)$ in the cefuroxime group $(\mathrm{P}=0.252$; table 3$)$. Of the 10 patients in the co-trimoxazole group with a clinically identifiable wound infection, eight had a positive bacterial culture, whereas the corresponding proportion in the cefuroxime group was 11 out of 14 patients. The most frequent type of bacteria detected in patients with an infection was Staphylococcus aureus.

The comparison groups showed no differences regarding changes in blood chemistry between baseline and follow-up (table 4).

\section{DISCUSSION}

This single centre, two arm, randomised, controlled, double blind clinical trial indicates that infection prophylaxis with $20 \mathrm{ml}$ co-trimoxazole, deposited in the PEG catheter immediately after its insertion, prevents wound infection at least as effectively as pre-operative intravenous cefuroxime in patients undergoing PEG.

Antibiotic prophylaxis is usually accomplished by using a second generation cephalosporin, such as cefuroxime, given as a single intravenous dose just before the PEG procedure. However, this strategy has several drawbacks. Firstly, PEG is a common procedure, and the cost of intravenous antibiotic drugs is not negligible. Secondly, giving an antibiotic intravenously is a time consuming process that requires special equipment. Thirdly, achieving the one hour time delay between the administration of the antibiotic and the insertion of the PEG catheter requires good collaboration between the hospital ward and the endoscopy unit. Fourthly, in the roughly $10 \%$ of cases where it proves impossible to insert a PEG catheter, the prophylaxis is given in vain. ${ }^{16}$ Fifthly, there is a risk of superimposed infection with Clostridium difficile after a single dose of a cephalosporin given as antibiotic prophylaxis. ${ }^{1718}$

Assuming that the results of the present study are true - that is, that co-trimoxazole solution given in the PEG catheter after insertion of the catheter can replace intravenous cephalosporin - these five problems are solved. This new strategy is inexpensive, requires no intravenous administration, and is given after the PEG catheter is in place. Another advantage of using the cotrimoxazole approach is that the treatment may potentially have a local antibacterial effect with rapid peritoneal absorption. ${ }^{1920}$

The efficacy of antibiotic prophylaxis in surgery has been shown to be reduced if it is administered more than one hour after incision, ${ }^{21}$ but the PEG procedure is very rapid re $^{22}$ so the time factor should not have a profound effect on the efficacy of co-trimoxazole prophylaxis. Co-trimoxazole, an inexpensive sulphonamide preparation combined with trimethoprim, in oral solution has good bioavailability and a long half life (about 10 hours for both components), and has an antibacterial spectrum suitable for the flora common in the upper gastrointestinal tract, including meticillin resistant $S$ aureus (MRSA). ${ }^{2324}$

\section{Comparison with other studies}

Although PEG is considered a relatively minor surgical procedure, it is associated with a general complications rate reported to be as high as $50 \%$ and a major complications rate of up to $7.4 \% .{ }^{1625}$ Wound infection is the most common problem, with a frequency of up to $32 \%$ without antibiotic prophylaxis. ${ }^{6-826}$ Such infections often require treatment with systemic antibiotics, intensive local wound care, and adjustment of the PEG catheter if fitted too tightly. These wound infections can also develop into a more devastating kind-for example, necrotising fasciitis - with high mortality. ${ }^{27}$ Infectious complications entail patient discomfort and costs; for example, owing to a longer hospital stay. ${ }^{28}$

Several meta-analyses have established that antibiotic prophylaxis in PEG procedures decreases the infection rate, with an absolute risk reduction of $14-17 \%$ and a

Table 3 | Bacterial growth in culture of samples retrieved from the percutaneous endoscopic gastrostomy (PEG) stoma at 7-14 days after insertion of the PEG catheter

\begin{tabular}{lcc} 
& $\begin{array}{c}\text { Co-trimoxazole } \\
(\mathrm{n}=116)\end{array}$ & $\begin{array}{c}\text { Cefuroxime } \\
(\mathrm{n}=118)\end{array}$ \\
Positive growth & $44(38)$ & $53(45)$ \\
\hline Negative growth & $37(32)$ & $31(26)$ \\
\hline No culture performed & $35(30)$ & $34(29)$ \\
\hline
\end{tabular}

Values are number (\%). 
Table 4 |Blood chemistry at baseline and at follow-up 7-14 days after insertion of the percutaneous endoscopic gastrostomy (PEG) catheter

\begin{tabular}{|c|c|c|c|c|}
\hline & \multicolumn{2}{|c|}{ Co-trimoxazole $(n=116)$} & \multicolumn{2}{|c|}{ Cefuroxime (n=118) } \\
\hline & Baseline & Follow-up & Baseline & Follow-up \\
\hline $\mathrm{C}$ reactive protein $(\mathrm{mg} / \mathrm{l})$ & $7.3(3.3,30.7)$ & $8.7(4.5,32.4)$ & $6.9(2.3,21.4)$ & $7.4(3.9,30.0)$ \\
\hline Haemoglobin (g/l) & $125(18)$ & $121(15)$ & $124(17)$ & $125(16)$ \\
\hline Albumin (g/l) & $32(27,36)$ & $32(27,35)$ & $33(28,36)$ & $33(29,36)$ \\
\hline
\end{tabular}

Values are median (lower and upper quartile) for $\mathrm{C}$ reactive protein, white blood cell count, and albumin. Values are mean ( \pm standard deviation) for haemoglobin.

number needed to treat to avoid one infection of between six and 10. ${ }^{1122930}$ To reduce infections and their negative effect on patients' quality of life, antibiotic prophylaxis during PEG is recommended. Our novel approach of giving an oral antibiotic solution directly into the PEG catheter immediately after insertion has not to our knowledge been tested previously.

Strengths and limitations of the study

Advantages of this study include the prospective randomised design, the blinding of all patients and evaluators, and the complementary use of objective markers of infections - that is, bacterial cultures and blood chemistry. Among the disadvantages, the included patients had to be well enough to be able to give informed consent, and, therefore, they might have been healthier than those who were not included. On the other hand, the patients with ear, nose, or throat cancer, who made up the majority of patients in both study groups, were likely to have received chemoradiotherapy, which could have a possible negative effect on their immunological defences.

The potential for problems with the subjectivity of infection diagnosis was counteracted by the strict blinding of patients and the experienced nurses who evaluated the patients at follow-up. Moreover, the secondary outcomes were objective and the results of the analysis of these outcomes supported the main finding.

\section{WHAT IS ALREADY KNOWN ON THIS TOPIC}

Wound infection is a common complication of percutaneous endoscopic gastrostomy (PEG)

Several randomised studies and meta-analyses have shown a positive effect of antibiotic prophylaxis on the risk of infection after insertion of a PEG catheter

Antibiotic prophylaxis usually comprises a single intravenous dose of a second generation cephalosporin administered just before the PEG procedure, which is rather expensive, time consuming, and can be given in vain in patients in whom the PEG procedure is impossible to accomplish

\section{WHAT THIS STUDY ADDS}

Administering the oral solution of co-trimoxazole, a combination of a sulphonamide and trimethoprim (Bactrim), in the PEG catheter immediately after insertion is as effective at preventing wound infections as the standard prophylaxis regimen of cefuroxime (Zinacef) given intravenously before the PEG procedure

This new strategy of antibiotic prophylaxis with co-trimoxazole can be administered rapidly and is inexpensive, safe, less likely to be administered needlessly, and could be used wherever in the world the PEG procedure is done

\section{Conclusions and policy implications}

In conclusion, this prospective clinical trial of patients who require PEG-which had a strict randomisation process and blinding of all patients and evaluators, and used several outcome markers - indicates that antibiotic prophylaxis with $20 \mathrm{ml}$ co-trimoxazole administered through a newly inserted PEG catheter prevents infectious complications at least as well as the standard prophylaxis with intravenous $1.5 \mathrm{~g}$ cefuroxime given before the PEG procedure.

We express our gratitude to Margrete Gellervik for excellent administrative help, to the nurses who cared for the patients with PEG catheters and managed all documentation, and the staff at the endoscopy unit.

Contributors: JL, PL, and LM formulated the study hypothesis. JB and JL recruited the patients and performed the percutaneous endoscopic gastrostomy procedures, and, together with PL and LM, met the patients at the initial visit. FM, JB, and JL did the statistical analyses. JB wrote the first draft of the manuscript. JL, PL, FM, and LM provided critical input at all stages and critically reviewed and contributed to the final draft. Funding: This study was funded by the Swedish Cancer Society and Swedish Research Council.

Competing interests: All authors have completed the Unified Competing Interest form at www.icmje.org/coi_disclosure.pdf (available on request from the corresponding author) and all authors declare: (1) No financial support for the submitted work from anyone other than their employer; (2) No financial relationships with commercial entities that might have an interest in the submitted work; (3) No spouses, partners, or children with relationships with commercial entities that might have an interest in the submitted work; (4) No non-financial interests that may be relevant to the submitted work.

Ethical approval: The regional ethics committee in Stockholm, Sweden, approved this study (registration number 05/505-31).

Data sharing: No additional data available.

1 Gauderer MW, Ponsky JL, Izant RJ Jr. Gastrostomy without laparotomy: a percutaneous endoscopic technique. J Pediatr Surg 1980;15:872-5.

2 Norman K, Pichard C, Lochs H, Pirlich M. Prognostic impact of disease-related malnutrition. Clin Nutr 2008;27:5-15.

3 McClave SA, Chang WK. Complications of enteral access. Gastrointest Endosc 2003;58:739-51.

4 Lee JH, Kim JJ, Kim YH, Jang JK, Son HJ, Peck KR, et al. Increased risk of peristomal wound infection after percutaneous endoscopic gastrostomy in patients with diabetes mellitus. Dig Liver Dis 2002:34:857-61.

5 Potack JZ, Chokhavatia S. Complications of and controversies associated with percutaneous endoscopic gastrostomy: report of a case and literature review. Medscape J Med 2008;10:142.

6 Luman W, Kwek KR, Loi KL, Chiam MA, Cheung WK, Ng HS. Percutaneous endoscopic gastrostomy-indications and outcome of our experience at the Singapore General Hospital. Singapore Med J 2001;42:460-5.

7 Gencosmanoglu R, Koc D, Tozun N. Percutaneous endoscopic gastrostomy: results of 115 cases. Hepatogastroenterology 2003;50:886-8.

8 Mahadeva S, Sam IC, Khoo BL, Khoo PS, Goh KL. Antibiotic prophylaxis tailored to local organisms reduces percutaneous gastrostomy site infection. Int J Clin Pract 2009;63:760-5.

9 Jafri NS, Mahid SS, Minor KS, Idstein SR, Hornung CA, Galandiuk S. Meta-analysis: antibiotic prophylaxis to prevent peristomal infection 
following percutaneous endoscopic gastrostomy. Aliment Pharmacol Ther 2007;25:647-56.

10 Sharma VK, Howden CW. Meta-analysis of randomized, controlled trials of antibiotic prophylaxis before percutaneous endoscopic gastrostomy. Am J Gastroenterol 2000;95:3133-6.

11 Lipp A, Lusardi G. Systemic antimicrobial prophylaxis for percutaneous endoscopic gastrostomy. Cochrane Database Syst Rev 2006;4:CD005571.

12 Lipp A, Lusardi G. A systematic review of prophylactic antimicrobials in PEG placement. J Clin Nurs 2009;18:938-48.

13 Allison MC, Sandoe JA, Tighe R, Simpson IA, Hall RJ, Elliott TS. Antibiotic prophylaxis in gastrointestinal endoscopy. Gut 2009;58:869-80.

14 Geroulanos S, Marathias K, Kriaras J, Kadas B. Cephalosporins in surgical prophylaxis. J Chemother 2001;13:23-6.

15 Mitchell NJ, Evans DS, Pollock D. Pre-operation single-dose cefuroxime antimicrobial prophylaxis with and without metronidazole in elective gastrointestinal surgery. J Antimicrob Chemother 1980;6:393-9.

16 Grant DG, Bradley PT, Pothier DD, Bailey D, Caldera S, Baldwin DL, et al. Complications following gastrostomy tube insertion in patients with head and neck cancer: a prospective multi-institution study, systematic review and meta-analysis. Clin Otolaryngol 2009;34:103-12.

17 Mukhtar S, Shaker H, Basarab A, Byrne JP. Prophylactic antibiotics and Clostridium difficile infection. J Hosp Infect 2006;64:93-4.

18 Carignan A, Allard C, Pepin J, Cossette B, Nault V, Valiquette L. Risk of Clostridium difficile infection after perioperative antibacterial prophylaxis before and during an outbreak of infection due to a hypervirulent strain. Clin Infect Dis 2008;46:1838-43.

19 Lee LH, Zaidman GW, Van Horn K. Topical bactrim versus trimethoprim and sulfonamide against nocardia keratitis. Cornea 2001;20:179-82.

20 Singlas E, Colin JN, Rottembourg J, Meessen JP, de Martin A, Legrain $M$, et al. Pharmacokinetics of sulfamethoxazoletrimethoprim combination during chronic peritoneal dialysis: effect of peritonitis. Eur J Clin Pharmacol 1982;21:409-15.
21 Burke JF. The effective period of preventive antibiotic action in experimental incisions and dermal lesions. Surgery 1961;50:161-8.

22 Hiki N, Maetani I, Suzuki Y, Washizawa N, Fukuda T, Yamaguchi T. Reduced risk of peristomal infection of direct percutaneous endoscopic gastrostomy in cancer patients: comparison with the pull percutaneous endoscopic gastrostomy procedure. J Am Coll Surg 2008;207:737-44.

23 Napolitano LM. Early appropriate parenteral antimicrobial treatment of complicated skin and soft tissue infections caused by methicillinresistant Staphylococcus aureus. Surg Infect (Larchmt) 2008;9(Suppl 1):17-27S.

24 Petri JWA. Sulfonamides, trimethoprim-sulfamethoxazole, quinolones, and agents for urinary tract infections. In: Brunton LL, Lazo JS, Parker KL, eds. Goodman \& Gilman's the pharmacological basis of therapeutics. McGraw-Hill, 2006.

25 Sheehan JJ, Hill AD, Fanning NP, Healy C, McDermott EW, O’Donoghue DP, et al. Percutaneous endoscopic gastrostomy: 5 years of clinical experience on 238 patients. Ir Med J 2003;96:265-7.

26 Grant JP. Percutaneous endoscopic gastrostomy. Initial placement by single endoscopic technique and long-term follow-up. Ann Surg 1993;217:168-74.

27 MacLean AA, Miller G, Bamboat ZM, Hiotis K. Abdominal wall necrotizing fasciitis from dislodged percutaneous endoscopic gastrostomy tubes: a case series. Am Surg 2004;70:827-31.

28 Kulling D, Sonnenberg A, Fried M, Bauerfeind P. Cost analysis of antibiotic prophylaxis for PEG. Gastrointest Endosc 2000;51:152-6.

29 Ahmad I, Mouncher A, Abdoolah A, Stenson R, Wright J, Daniels A, et al. Antibiotic prophylaxis for percutaneous endoscopic gastrostomy-a prospective, randomised, double-blind trial. Aliment Pharmacol Ther 2003;18:209-15.

30 Saadeddin A, Freshwater DA, Fisher NC, Jones BJ. Antibiotic prophylaxis for percutaneous endoscopic gastrostomy for nonmalignant conditions: a double-blind prospective randomized controlled trial. Aliment Pharmacol Ther 2005;22:565-70.

Accepted: 22 April 2010 